\section{Violência doméstica e familiar contra a mulher: estudo de casos e controles com vítimas atendidas em serviços de urgência e emergência}

\author{
Domestic and family violence against women: \\ a case-control study with victims treated in \\ emergency rooms
}

\author{
La violencia doméstica y familiar contra la mujer: \\ estudio de casos y controles con víctimas \\ tratadas en salas de emergencia
}

\begin{abstract}
Resumo
O objetivo deste estudo foi identificar fatores associados ao atendimento por violência doméstica e familiar entre vítimas atendidas em serviços de urgência e emergência no Brasil. Realizou-se estudo de casos e controles baseado no Sistema de Vigilância de Violências e Acidentes (VIVA), 2011. Foram selecionadas mulheres com 18 anos ou mais de idade, vítimas de violência doméstica e familiar (casos) em comparação com aquelas vítimas de acidentes (controles). As razões de chances ajustadas foram estimadas por regressão logística não condicional. Foram incluídos 623 casos e 10.120 controles. Na análise ajustada, foram fatores de risco: idade mais jovem (18-29 anos), baixa escolaridade, não exercer atividade remunerada, consumo de bebida alcoólica, procura de atendimento em outro serviço, ocorrência em final de semana e durante a noite ou madrugada. A violência doméstica e familiar teve o consumo de bebida alcoólica como fator fortemente associado. Os dias e horas de maior ocorrência evidenciam a necessidade de adequação dos serviços de atendimento às vítimas.
\end{abstract}

Serviços Médicos de Emergência; Violência contra a Mulher;

Violência Doméstica; Saúde da Mulher; Estudos de Casos e Controles
Leila Posenato Garcia 1

Elisabeth Carmen Duarte 2

Lúcia Rolim Santana de Freitas 3

Gabriela Drummond Marques da Silva 4

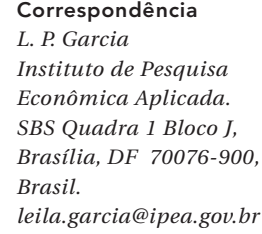

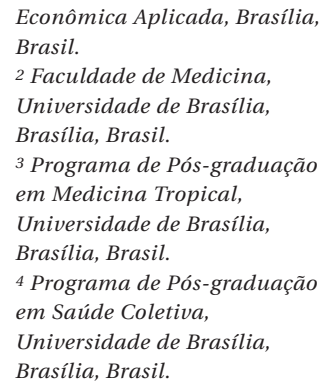




\section{Introdução}

A violência contra a mulher, além de uma violação aos direitos humanos, é um importante problema de saúde pública. A violência física e sexual contra as mulheres resulta em expressivos custos econômicos e sociais, pode gerar graves consequências para a saúde mental e reprodutiva, assim como afetar também as crianças e o ambiente familiar 1

Segundo a Organização Mundial da Saúde (OMS), a prevalência global de violência física e/ou sexual contra a mulher perpetrada por parceiro íntimo foi de 30\% (intervalo de $95 \%$ de confiança - IC95\%: 27,8\%-32,2\%). As mulheres residentes nos países da África, Oriente Médio e Sudeste da Ásia reportaram as prevalências mais elevadas (em torno de $37 \%$ ), seguidas por aquelas das Américas (aproximadamente 30\%) 1.

No Brasil, o estudo multicêntrico sobre saúde da mulher e violência doméstica, coordenado pela OMS (WHO Multi-country Study on Women's Health and Domestic Violence), foi realizado em São Paulo e Pernambuco, no período 2000-2001. Em São Paulo, 41,8\% das mulheres relataram ao menos uma vez na vida terem sofrido violência psicológica, $27,2 \%$ física e $10,1 \%$ sexual. Em Pernambuco, essas prevalências foram ainda mais elevadas, correspondendo a 48,9\%, 33,7\% e $14,3 \%$, respectivamente 2 .

Uma pesquisa encomendada pela Secretaria de Políticas para as Mulheres, da Presidência da República do Brasil, em 2013, revelou que 54\% dos entrevistados declararam conhecer pelo menos uma mulher que havia sido agredida por um parceiro e $56 \%$ afirmaram conhecer pelo menos um homem que havia agredido uma parceira. Ainda, $89 \%$ e $88 \%$ dos entrevistados consideram que, nos últimos cinco anos, houve aumento, respectivamente, da ocorrência de agressões e assassinatos de mulheres por parceiros ou exparceiros ${ }^{3}$. A pesquisa do Instituto Avon e do Datapopular, realizada no mesmo ano, revelou que $56 \%$ dos homens admitiram que já haviam cometido alguma ação considerada violência contra a mulher, como xingar, empurrar, humilhar em público ou ameaçar com palavras 4 .

A violência doméstica e familiar contra a mulher é um problema de grande magnitude no Brasil. Dentre as notificações ao Sistema de Vigilância de Violências e Acidentes (VIVA) em 2008, em 75,9\% dos casos de violência contra a mulher os agressores foram familiares e conhecidos. Ademais, 39,7\% das mulheres indicaram ter sofrido violência de repetição, enquanto que, entre os homens, esta proporção foi $26,3 \% 5$.

Em 2006, foi sancionada a Lei no 11.340, conhecida como Lei Maria da Penha, que criou mecanismos para coibir a violência doméstica e familiar contra a mulher no Brasil 6. Todavia, poucos estudos investigaram os fatores associados a esse tipo de violência no país $7,8,9$. O conhecimento desses fatores pode ser útil para subsidiar políticas e programas voltados a seu enfrentamento.

O presente estudo tem como objetivo analisar os fatores associados ao atendimento por violência doméstica e familiar entre vítimas de acidentes e violências atendidas em serviços de urgência e emergência, participantes do inquérito que compõe o VIVA, realizado em 2011.

\section{Métodos}

Foi realizado um estudo de casos e controles com base nos dados das vítimas de acidentes e violências, coletados por meio de inquérito que compõe o VIVA em 2011.

Os serviços de urgência e emergência vinculados ao Sistema Único de Saúde (SUS) foram selecionados com base no Cadastro Nacional de Estabelecimentos de Saúde (CNES), tendo como critérios de inclusão: (1) prestar serviço de urgência e emergência e (2) ser serviço de referência para o atendimento de causas externas no município, segundo indicação das respectivas secretarias municipais de saúde. O tamanho mínimo da amostra calculado para o inquérito foi de 2.000 atendimentos pelas causas selecionadas (acidentes e violências) em cada uma das capitais estudadas 10 .

$\mathrm{O}$ inquérito foi realizado nos serviços situados em 24 capitais brasileiras e no Distrito Federal. As capitais dos estados do Amazonas (Manaus) e São Paulo (São Paulo) foram excluídas devido a questões relacionadas a aspectos técnico-operacionais e de gestão local, que resultaram em perda de informação ${ }^{10}$. O inquérito abrangeu 71 serviços, todavia, para o propósito do presente trabalho foram excluídos dois serviços nos quais foram registrados exclusivamente atendimentos de crianças. Assim, para este estudo foram considerados os dados coletados em 69 serviços.

Os dados do inquérito VIVA foram coletados em um período de 30 dias consecutivos em cada um dos serviços participantes, divididos em 60 turnos de 12 horas, entre os meses de setembro e outubro de 2011. Para a obtenção do número de turnos sorteados em cada serviço, o tamanho mínimo da amostra (2.000) foi dividido pelo número médio por turno de atendimentos por causas externas realizadas no mesmo serviço em anos anteriores. Foram incluídos na amostra todos os atendimentos por acidentes e violências 
realizados nos turnos sorteados. Mais informações sobre o inquérito podem ser obtidas em publicação específica 10.

Com base no VIVA, foi delineado um estudo de casos e controles. O grupo caso incluiu as mulheres com 18 anos de idade ou mais, vítimas de violência doméstica e familiar, que foram atendidas nos serviços e turnos selecionados. A definição de violência doméstica adotada é aquela constante na Lei Maria da Penha 6: “...qualquer ação ou omissão baseada no gênero que lhe cause morte, lesão, sofrimento físico, sexual ou psicológico e dano moral ou patrimonial: I - no âmbito da unidade doméstica, compreendida como o espaço de convívio permanente de pessoas, com ou sem vínculo familiar, inclusive as esporadicamente agregadas; II - no âmbito da família, compreendida como a comunidade formada por indivíduos que são ou se consideram aparentados, unidos por laços naturais, por afinidade ou por vontade expressa; III - em qualquer relação íntima de afeto, na qual o agressor conviva ou tenha convivido com a ofendida, independentemente de coabitação".

Assim, consideraram-se casos todas as mulheres atendidas cujo tipo de ocorrência foi agressão/maus-tratos, tendo como provável autor da agressão mãe/pai, companheiro(a) ou ex-companheiro(a), outro familiar e amigo/conhecido. Consideraram-se controles todas as mulheres da mesma faixa de idade atendidas nos mesmos serviços e turnos dos casos, que foram vítimas de causas acidentais (acidentes de transporte, quedas, queimaduras e outros acidentes). As vítimas de causas externas autoprovocadas, geradas por intervenção legal ou por agente desconhecido ou não declarado, não foram incluídas no estudo.

Os casos foram caracterizados considerandose as seguintes variáveis:

- Provável autor da agressão, referido pela mulher (companheiro/ex-companheiro, amigo/conhecido, pai/mãe, outro familiar);

- Natureza da agressão (física, sexual, psicológica/abandono/negligência);

- Meio de agressão (força corporal/espancamento, objeto perfurocortante, objeto contundente, arma de fogo, ameaça, outros/ignorado);

- Sexo do provável autor da agressão (masculino, feminino, ambos os sexos, não informado);

- Local de ocorrência (residência, via pública, bar ou similar, outro);

- Natureza da lesão (corte/laceração, contusão, traumatismo cranioencefálico/politraumatismo, entorse/luxação, fratura, sem lesão, outros/não informado);

- Parte do corpo atingida (cabeça, membros superiores, múltiplos órgãos, tórax/dorso, membros inferiores, boca/dentes, outros, sem lesão, não informado);

- Evolução na emergência nas primeiras 24 horas (alta, internação hospitalar, encaminhamento ambulatorial, encaminhamento para outro serviço, evasão/fuga, óbito, ignorado);

- Hora da ocorrência.

As variáveis independentes investigadas para casos e controles foram:

- Idade (18-29; 30-44; 45-49 ou $\geq 60$ anos);

- Cor ou raça (branca, preta/parda ou amarela/ indígena);

- Escolaridade ( $>8$ ou $\leq 8$ anos de estudo);

- Atividade remunerada atual (sim ou não);

- Consumo de bebida alcoólica pela vítima nas 6 horas anteriores à ocorrência, referido pela entrevistada, ou suspeitado pelo entrevistador (sim ou não);

- Procura de atendimento em outro serviço pela mesma ocorrência, antes do atendimento no local da entrevista (sim ou não);

- Dia da semana da ocorrência (segunda a sexta, sábado ou domingo);

- Período/turno da ocorrência (manhã, tarde, noite ou madrugada).

Foram calculadas as frequências absolutas e relativas das categorias das variáveis. A análise foi conduzida por meio de regressão logística não condicional bivariável e múltipla, para estimar as razões de chances (RC) brutas e ajustadas e seus respectivos IC95\%, respectivamente. Na análise ajustada, foram incluídas as variáveis com valor de $\mathrm{p}<0,20$ na análise bivariável. Foi considerado o nível de significância estatística de 5\%. As análises foram realizadas com o auxílio do software Stata, versão 12 (StataCorp LP, College Station, Estados Unidos).

O inquérito VIVA foi aprovado pela Comissão Nacional de Ética em Pesquisa (CONEP) do Ministério da Saúde, sob Parecer de Emenda no 006/2011. Obteve-se o consentimento verbal dos participantes, por tratar-se de ação de vigilância em saúde de âmbito nacional. A base de dados utilizada no presente trabalho não continha a identificação dos participantes e foi cedida mediante autorização do Departamento de Vigilância de Doenças e Agravos Não Transmissíveis e Promoção da Saúde, Secretaria de Vigilância em Saúde, Ministério da Saúde.

\section{Resultados}

Um total de 16.256 mulheres demandaram os serviços selecionados no VIVA, sendo que destas, $5.104(31,4 \%)$ eram menores de 18 anos de idade (Figura 1). Entre as 11.152 mulheres com 18 anos ou mais, 409 (3,7\%) foram excluídas por não 
Fluxograma do estudo de casos e controles sobre a ocorrência de violência doméstica e familiar entre mulheres com idade $\geq 18$ anos, atendidas em serviços de urgência e emergência. Inquérito VIVA, 2011.

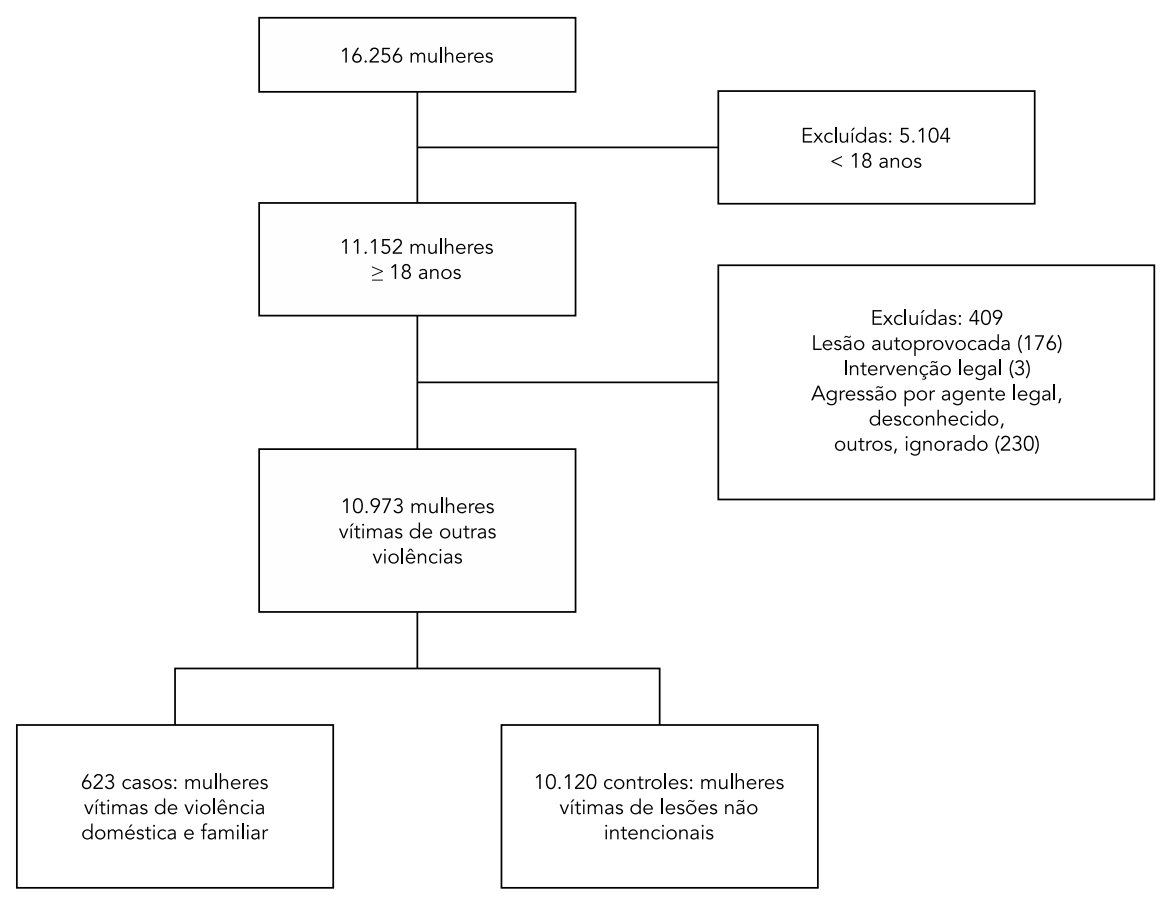

atenderem aos critérios de definição de casos e controles adotados no presente estudo (Figura 1). Dessa forma, foram incluídos 623 casos (vítimas de violência doméstica ou familiar) e 10.120 controles (vítimas de causas acidentais), com uma razão controles/casos de 16,1.

As características das agressões sofridas pelas vítimas de violência doméstica e familiar (casos) estão descritas na Tabela 1. Quase a totalidade sofreu violência física $(97,4 \%)$, tendo como meios de agressão predominantes a força corporal/ espancamento (60,7\%), objeto perfurocortante (20,9\%) e objeto contundente (11,9\%). Entre os prováveis autores das agressões, predominaram os companheiros ou ex-companheiros $(51,5 \%)$ e indivíduos do sexo masculino $(74,1 \%)$. O local mais frequente da ocorrência foi a residência (63,6\%). Quanto à natureza da lesão, predominaram corte/laceração $(46,4 \%)$ e contusão (20,2\%). As partes do corpo mais frequentemente atingidas foram a cabeça $(39,5 \%)$, seguida por membros superiores $(21,8 \%)$ e múltiplos órgãos (13,9\%). A maioria dos atendimentos evoluiu pa- ra alta $(75 \%), 8,8 \%$ requereram internação hospitalar e $0,7 \%$ evoluiu para óbito.

A Figura 2 ilustra o número de atendimentos, segundo hora da ocorrência do episódio de violência. É evidente a concentração dos casos no período das 18 às 24 horas.

O contraste entre casos e controles é apresentado na Tabela 2. Quanto à idade, predominaram mulheres jovens, $46,5 \%$ dos casos e $32,3 \%$ dos controles tinham de 18 a 29 anos de idade; $71,5 \%$ dos casos e $62,6 \%$ dos controles eram pretas ou pardas; $53,8 \%$ dos casos e $45,6 \%$ dos controles tinham até 8 anos de estudo; $51,5 \%$ dos casos e $47,9 \%$ dos controles não tinham atividade remunerada; $32 \%$ dos casos e 5,9\% dos controles tinham relato de consumo de bebida alcoólica nas seis horas anteriores à ocorrência; $26,5 \%$ dos casos e 22,5\% dos controles haviam procurado atendimento em outro serviço; $46,1 \%$ dos casos e $30,5 \%$ dos controles foram atendidos nos finais de semana. Entre os casos, $58,2 \%$ dos atendimentos ocorreram durante a noite ou madrugada, enquanto entre os controles esta proporção foi $36,5 \%$. 
Tabela 1

Descrição das características das agressões sofridas por mulheres com idade $\geq 18$ anos, vítimas de violência doméstica e familiar, atendidas em serviços de urgência e emergência. Inquérito VIVA, 2011.

\begin{tabular}{|c|c|c|}
\hline Variáveis & $\mathbf{n}$ & $\%$ \\
\hline \multicolumn{3}{|l|}{ Natureza da agressão } \\
\hline Física & 607 & 97,4 \\
\hline Sexual & 2 & 0,3 \\
\hline Psicológica/Abandono/Negligência & 14 & 2,3 \\
\hline \multicolumn{3}{|l|}{ Meio de agressão } \\
\hline Força corporal/Espancamento & 377 & 60,7 \\
\hline Objeto perfurocortante & 130 & 20,9 \\
\hline Objeto contundente & 74 & 11,9 \\
\hline Arma de fogo & 15 & 2,4 \\
\hline Ameaça & 10 & 1,6 \\
\hline Outros/lgnorado & 15 & 2,5 \\
\hline \multicolumn{3}{|l|}{ Provável autor da agressão } \\
\hline Companheiro/Ex-companheiro & 321 & 51,5 \\
\hline Amigo/Conhecido & 172 & 27,6 \\
\hline Outro familiar & 117 & 18,8 \\
\hline Pai/Mãe & 13 & 2,1 \\
\hline \multicolumn{3}{|l|}{ Sexo do provável autor da agressão } \\
\hline Masculino & 461 & 74,1 \\
\hline Feminino & 145 & 23,3 \\
\hline Ambos os sexos & 10 & 1,6 \\
\hline Não informado & 6 & 1,0 \\
\hline \multicolumn{3}{|l|}{ Local de ocorrência } \\
\hline Residência & 389 & 63,6 \\
\hline Via pública & 137 & 22,4 \\
\hline Bar ou similar & 54 & 8,8 \\
\hline Outro & 32 & 5,2 \\
\hline \multicolumn{3}{|l|}{ Natureza da lesão } \\
\hline Corte/Laceração & 289 & 46,4 \\
\hline Contusão & 126 & 20,2 \\
\hline Traumatismo cranioencefálico/Politraumatismo & 49 & 7,9 \\
\hline Entorse/Luxação & 45 & 7,2 \\
\hline Fratura & 44 & 7,1 \\
\hline Sem lesão & 32 & 5,1 \\
\hline Outros/Não informado & 38 & 6,1 \\
\hline \multicolumn{3}{|l|}{ Parte do corpo atingida * } \\
\hline Cabeça/Pescoço & 246 & 39,5 \\
\hline Membros superiores & 136 & 21,8 \\
\hline Múltiplos órgãos & 87 & 13,9 \\
\hline Tórax/Dorso/Abdome/Quadril & 55 & 8,8 \\
\hline Membros inferiores & 32 & 5,1 \\
\hline Boca/Dentes & 23 & 3,7 \\
\hline Sem lesão & 32 & 5,1 \\
\hline Outros/Não informado & 12 & 2,1 \\
\hline \multicolumn{3}{|l|}{ Evolução na emergência nas primeiras 24 horas } \\
\hline Alta & 463 & 75,0 \\
\hline Internação hospitalar & 54 & 8,8 \\
\hline Encaminhamento ambulatorial & 36 & 5,8 \\
\hline Encaminhamento para outro serviço & 33 & 5,4 \\
\hline Evasão/Fuga & 20 & 3,2 \\
\hline Óbito & 4 & 0,7 \\
\hline Ignorado & 7 & 1,1 \\
\hline
\end{tabular}

* Diagnóstico principal. 
Número de casos segundo hora de ocorrência * da violência entre mulheres vítimas de violência doméstica e familiar com idade $\geq 18$ anos, atendidas em serviços de urgência e emergência. Inquérito VIVA, 2011.

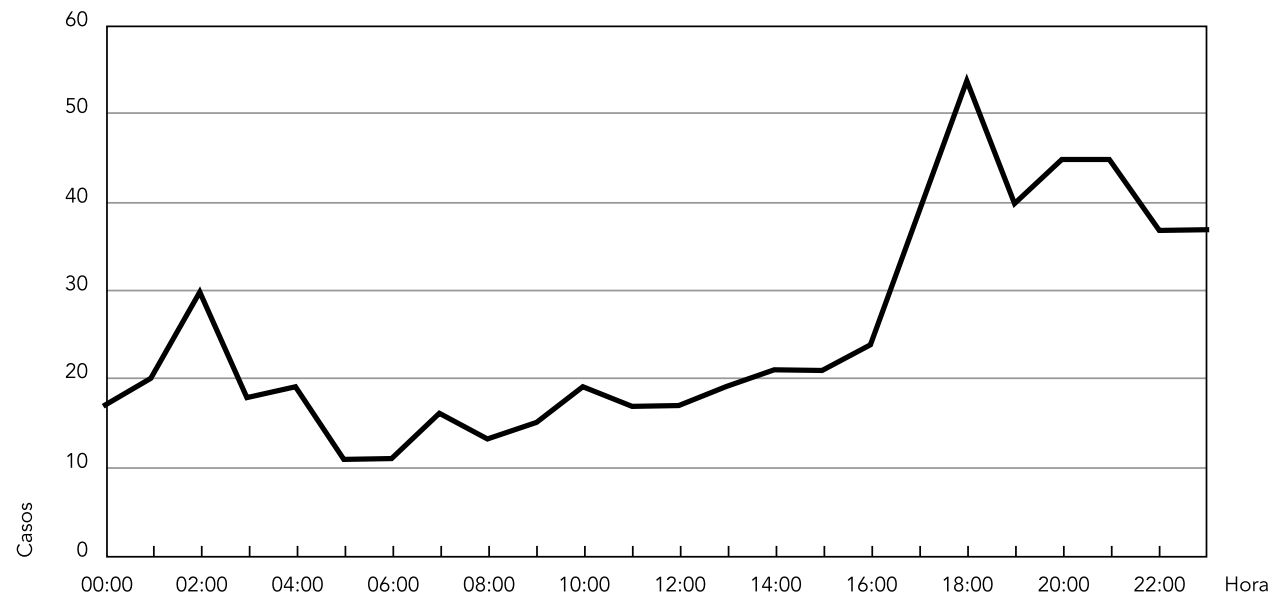

* 605 dos 623 casos registraram a hora da ocorrência.

Na análise bruta, todas as variáveis apresentaram associação estatisticamente significativa $(\mathrm{p}<0,05)$ com violência doméstica e familiar, à exceção de exercer atividade remunerada $(\mathrm{p}=$ 0,089 ). Como definido na metodologia, todas as variáveis independentes que apresentaram associação no nível de significância de $\mathrm{p}<0,20$ foram incluídas no modelo multivariável (Tabela 2 ).

$\mathrm{Na}$ análise ajustada, a variável exercer atividade remunerada passou a apresentar significância estatística ( $p=0,002)$, e a cor da pele perdeu significância $(p=0,104)$. Foram fatores associados à maior chance de atendimento por violência doméstica e familiar contra a mulher: idade jovem (18-29 anos); escolaridade de até 8 anos de estudos ( $\mathrm{RC}=1,96$; IC95\%: 1,62-2,37); não exercer atividade remunerada $(\mathrm{RC}=1,34$; IC95\%: 1,12-1,61); consumo de bebida alcoólica ( $\mathrm{RC}=4,49$; IC95\%: 3,59-5,59); procura de atendimento em outro serviço ( $\mathrm{RC}=1,28$; IC95\%: $1,05-1,57)$; ocorrência em final de semana (RC = 1,33; IC95\%: 1,10-1,59); e ocorrência durante a noite ( $\mathrm{RC}=2,02$; IC95\%: 1,57-2,61) ou madrugada (RC = 2,82; IC95\%: 2,06-3,88), em comparação com a manhã.

\section{Discussão}

Este estudo é o primeiro a avaliar fatores associados ao atendimento de mulheres vítimas por violência doméstica e familiar em serviços de urgência e emergência incluindo 25 capitais brasileiras. Os fatores associados a esses atendimentos foram: idade jovem, menor escolaridade não exercer atividade remunerada, consumo de bebida alcoólica pela vítima, procura de atendimento em outro serviço, ocorrência durante o final de semana, bem como nos períodos da noite e madrugada.

Os achados do presente trabalho foram consistentes com aqueles de outros estudos realizados no Brasil e no exterior 7,8,9,11,12. O consumo de bebida alcoólica pela vítima foi o fator mais fortemente associado ao atendimento resultante de violência doméstica e familiar contra a mulher neste trabalho. A relação entre consumo abusivo de álcool e violência doméstica é amplamente conhecida e documentada 13. O estudo transversal realizado em um município paulista revelou risco duas vezes e meia maior de sofrer violência por parceiro íntimo para as mulheres que relataram ter realizado uso de álcool 9 .

Ademais, a associação entre violência doméstica e familiar e uso de álcool pela mulher pode ser confundida ou potencializada pelo uso de álcool pelo agressor 11 que, todavia, não pôde ser investigado no presente estudo, realizado com dados secundários. Um estudo realizado nos Estados Unidos revelou que o uso de álcool pelo agressor foi associado ao aumento de oito vezes 
Efeitos brutos e ajustados das variáveis independentes do estudo sobre a ocorrência de violência doméstica e familiar entre mulheres com idade $\geq 18$ anos, atendidas em serviços de urgência e emergência. Inquérito VIVA, 2011.

\begin{tabular}{|c|c|c|c|c|c|c|c|c|c|c|}
\hline \multirow[t]{2}{*}{ Variáveis } & \multicolumn{2}{|c|}{$\begin{array}{l}\text { Casos } \\
(\mathrm{N}=623)\end{array}$} & \multicolumn{2}{|c|}{$\begin{array}{l}\text { Controles } \\
(\mathrm{N}=10.120)\end{array}$} & \multirow[b]{2}{*}{$\begin{array}{c}\mathrm{RC} \\
\text { bruta }\end{array}$} & \multicolumn{2}{|c|}{ Análise bruta } & \multicolumn{3}{|c|}{ Análise ajustada * } \\
\hline & n & $\%$ & $\mathrm{n}$ & $\%$ & & $\mathrm{IC} 95 \%$ & $\begin{array}{l}\text { Valor de } \\
p \text { ** }\end{array}$ & $\begin{array}{c}\mathrm{RC} \\
\text { ajustada }\end{array}$ & IC95\% & $\begin{array}{l}\text { Valor de } \\
\text { p ** }\end{array}$ \\
\hline Idade (anos) & & & & & & & $<0,001$ & & & $<0,001$ \\
\hline $18-29$ & 290 & 46,5 & 3.265 & 32,3 & 1,00 & & & 1,00 & & \\
\hline $30-44$ & 229 & 36,8 & 3.030 & 29,9 & 0,85 & $0,71-1,02$ & & 0,79 & $0,64-0,97$ & \\
\hline $45-49$ & 79 & 12,7 & 2.062 & 20,4 & 0,43 & $0,33-0,56$ & & 0,41 & $0,31-0,54$ & \\
\hline$\geq 60$ & 25 & 4,0 & 1.763 & 17,4 & 0,16 & $0,11-0,24$ & & 0,13 & $0,08-0,21$ & \\
\hline Cor ou raça & & & & & & & $<0,001$ & & & 0,104 \\
\hline Branca & 165 & 26,6 & 3.498 & 34,6 & 1,00 & & & 1,00 & & \\
\hline Preta/Parda & 444 & 71,5 & 6.318 & 62,6 & 1,49 & $1,24-1,79$ & & 1,15 & $0,94-1,41$ & \\
\hline Amarela/Indígena & 12 & 1,9 & 281 & 2,8 & 0,75 & $0,49-1,65$ & & 0,67 & $0,36-1,25$ & \\
\hline Escolaridade (anos de estudo) & & & & & & & $<0,001$ & & & $<0,001$ \\
\hline$>8$ & 268 & 46,2 & 5.288 & 54,4 & 1,00 & & & 1,00 & & \\
\hline$\leq 8$ & 312 & 53,8 & 4.439 & 45,6 & 1,39 & $1,17-1,65$ & & 1,96 & $1,62-2,37$ & \\
\hline Atividade remunerada & & & & & & & 0,087 & & & 0,002 \\
\hline Sim & 294 & 48,5 & 5.176 & 52,1 & 1,00 & & & 1,00 & & \\
\hline Não & 312 & 51,5 & 4.761 & 47,9 & 1,15 & $0,98-1,36$ & & 1,34 & $1,12-1,61$ & \\
\hline 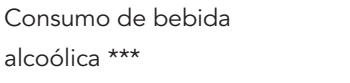 & & & & & & & $<0,001$ & & & $<0,001$ \\
\hline Não & 417 & 68,0 & 9.467 & 94,1 & 1,00 & & & 1,00 & & \\
\hline Sim & 196 & 32,0 & 592 & 5,9 & 7,52 & $6,19-9,12$ & & 4,49 & $3,59-5,59$ & \\
\hline $\begin{array}{l}\text { Procura de atendimento em } \\
\text { outro serviço \# }\end{array}$ & & & & & & & 0,025 & & & 0,015 \\
\hline Não & 453 & 73,5 & 7.747 & 77,5 & 1,00 & & & 1,00 & & \\
\hline Sim & 163 & 26,5 & 2.249 & 22,5 & 1,24 & $1,03-1,49$ & & 1,28 & $1,05-1,57$ & \\
\hline Dia da semana da ocorrência & & & & & & & $<0,001$ & & & 0,003 \\
\hline Segunda a sexta & 334 & 53,9 & 7.002 & 69,5 & 1,00 & & & 1,00 & & \\
\hline Sábado ou domingo & 286 & 46,1 & 3.074 & 30,5 & 1,95 & $1,65-2,30$ & & 1,33 & $1,10-1,59$ & \\
\hline Período da ocorrência & & & & & & & $<0,001$ & & & $<0,001$ \\
\hline Manhã & 104 & 16,7 & 2.958 & 29,3 & 1,00 & & & 1,00 & & \\
\hline Tarde & 156 & 25,1 & 3.451 & 34,2 & 1,29 & $0,99-1,65$ & & 1,27 & $0,96-1,66$ & \\
\hline Noite & 252 & 40,5 & 3.033 & 30,1 & 2,36 & $1,87-2,99$ & & 2,02 & $1,57-2,61$ & \\
\hline Madrugada & 110 & 17,7 & 650 & 6,4 & 4,81 & $3,63-6,38$ & & 2,82 & $2,06-3,88$ & \\
\hline
\end{tabular}

IC95\%: intervalo de 95\% de confiança; RC: razão de chances.

* Ajustada por idade, cor ou raça, escolaridade, atividade remunerada, consumo de bebida alcoólica, procura de atendimento em outro serviço,

dia da semana da ocorrência e período da ocorrência;

** Teste qui-quadrado;

*** Nas 6 horas anteriores, referido pela entrevistada, ou suspeitado pelo entrevistador;

\# Pela mesma ocorrência, antes do atendimento no local da entrevista.

na ocorrência de abuso à mulher, após ajuste para as variáveis demográficas ${ }^{12}$. Em um estudo transversal com usuárias do SUS de Campinas (São Paulo), foi revelado que o uso de álcool pelo parceiro esteve associado à violência doméstica na gravidez ${ }^{7}$. Contudo, aparentemente o uso do álcool, tanto pela vítima como pelo agressor ou por ambos, é apenas um fator contribuinte em uma complexa rede causal de determinação da violência doméstica e familiar. É amplamente conhecido que a violência doméstica e familiar contra a mulher é de natureza multicausal 
e inclui fatores sociais, culturais, familiares, da relação entre os parceiros e individuais 14 .

Entre as variáveis estudadas, ficou evidente a associação do atendimento por violência doméstica e familiar contra a mulher com a pior condição socioeconômica da vítima, mensurada pela menor escolaridade e não exercício de atividade remunerada. De maneira consistente, um estudo transversal realizado em São Paulo e Pernambuco identificou a menor escolaridade como fator de risco para a violência por parceiro íntimo ${ }^{8}$.

Após análise ajustada, este estudo não identificou associação com a cor da pele, o que também é consistente com o estudo previamente citado ${ }^{8}$, cujas autoras sugerem que a maior prevalência de violência por parceiro íntimo na vida entre mulheres negras possa estar associada a condições socioeconômicas desfavoráveis. No entanto, as mesmas autoras indicam a realização de estudos para investigar com maior profundidade o significado da cor ou raça na violência doméstica 8 .

Neste trabalho, a faixa etária de 18 a 29 anos foi a de maior chance para atendimento decorrente de violência doméstica e familiar contra a mulher. Em outros estudos realizados no Brasil, a idade não esteve associada à ocorrência de violência por parceiro íntimo ${ }^{8,9}$. Todavia, há que se destacar que esses estudos consideraram a ocorrência de violência na vida, enquanto o presente estudo considerou o episódio que gerou a necessidade atual de atendimento. De forma semelhante ao presente trabalho, estudos que consideraram a violência ocorrida no último ano também encontraram associação com idades mais jovens. Uma hipótese levantada para explicar esses achados é que, à medida que as mulheres envelhecem elas se tornam economicamente mais produtivas e socialmente influentes, ficando assim menos propensas a registrar violência recente do que as mulheres mais jovens 14,15. Dessa forma, mesmo que já tenham sido expostas à situação de violência doméstica durante a juventude, as mulheres mais velhas podem ter tido a oportunidade de se desvencilhar desta condição e não buscam mais atendimento por este motivo, favorecendo a associação do atendimento atual por esta causa com as idades mais jovens.

Outros achados deste estudo são particularmente relevantes para subsidiar políticas públicas voltadas ao enfrentamento da violência doméstica e familiar contra a mulher. A ocorrência durante os finais de semana, bem como nos períodos da noite e madrugada foram fatores associados ao atendimento. Esses achados reforçam a importância de que os serviços voltados ao atendimento às mulheres vítimas de violência funcionem em horários diferenciados, justamente para cobrir os períodos de maior demanda - finais de semana, noites e madrugadas. Vale destacar que são nesses dias e horários que o contato entre agressor e vítima ocorre de maneira mais continuada, intensificam-se as possibilidades de consumo de álcool, que poderá interagir com os demais determinantes da violência, e ampliamse as possibilidades de eventos sociais que podem favorecer os episódios de violência. Esses fatores devem ser mais bem investigados em trabalhos futuros.

Outro achado igualmente relevante para as políticas de saúde foi a associação com a procura de atendimento em outro serviço, pela mesma ocorrência, antes do atendimento no local da entrevista. Esse resultado sugere que muitas mulheres vítimas de violência doméstica ou familiar não tiveram o acolhimento adequado no primeiro local que buscaram, o que pode indicar despreparo dos serviços para o atendimento a estas vítimas, e/ou a necessidade de encaminhamento para outro serviço de saúde.

A OMS reforça a necessidade de melhorar a capacidade dos serviços de saúde para identificarem a violência doméstica contra a mulher e o risco de feminicídio 16 . O encaminhamento adequado e oportuno da mulher agredida, e seu acesso aos serviços de segurança pública e justiça para a obtenção de medidas protetivas podem fazer a diferença entre a vida e a morte. Nesse sentido, alguns estados brasileiros, a exemplo do Rio Grande do Sul, adotaram um serviço conhecido como "Patrulha Maria da Penha". Trata-se de patrulhas que atuam com viaturas exclusivamente destinadas à realização de visitas domiciliares rotineiras e coordenadas, com o objetivo de atuar de forma preventiva junto a situações nas quais já tenham sido emitidas as medidas protetivas em defesa de mulheres vítimas de agressões. Com base nos resultados deste estudo, recomenda-se que a atividade das patrulhas seja intensificada durante os dias e horários identificados como de maior risco, e que as causas de busca de mais de um serviço de saúde para atendimento dessa natureza sejam mais estudadas.

Este estudo apresenta algumas limitações que merecem atenção. Com respeito à validade externa, é importante destacar que a população de estudo refere-se a mulheres atendidas em serviços de urgência e emergência do SUS nos locais e períodos de coleta de dados do inquérito VIVA. Dessa forma, os achados do presente trabalho referem-se a essa população fonte, e não podem ser generalizados à população de mulheres vítimas de violência doméstica e familiar do Brasil, muitas das quais não buscam atendimento e/ou residem em áreas não cobertas pelo VIVA. Outra 
possível fonte de limitação para a generalização do estudo é o encaminhamento de determinados perfis de vítimas para determinados serviços de saúde (referência diferencial). Quando isso acontece, um fator pode ser de risco quando investigado em um serviço, e de proteção em outro. A coleta de dados em 69 serviços de 25 capitais do Brasil possivelmente minimizou essa limitação.

Com respeito a erros de mensuração, por razões evidentes, as variáveis estudadas se limitaram àquelas presentes no inquérito VIVA. Esse fato impediu a inclusão de variáveis importantes para a análise, tais como história de violência pregressa e detalhamentos das características do agressor 11. Adicionalmente, o desfecho foi construído com base em informações referidas pela vítima ou acompanhante, ou ainda pelo entrevistador. Isso pode ter gerado erro de classificação de casos como sendo controles. Por exemplo, vítimas de violência doméstica e familiar sendo identificadas como vítimas de queda, e assim incluídas no grupo controle. Esse tipo de erro, se não diferencial quanto às características de exposição, pode ter gerado subestimativa das medidas de efeito.

Ainda, foi considerada a violência doméstica e familiar em seu sentido mais amplo, não restrito à violência por parceiro íntimo. Essas características do presente estudo limitam a sua comparabilidade com outros trabalhos sobre o tema. No que se refere ao viés de sobrevivência, vale destacar que neste estudo foram incluídas apenas as vítimas que chegaram vivas ao serviço e receberam atendimento. Assim, o estudo não necessariamente revela os fatores que influenciaram a chance de sofrer violência doméstica, mas sim de sofrer violência doméstica e sobreviver a ela.

Além disso, merecem menção algumas das limitações específicas dos estudos de casos e controles 17 . O viés de memória, embora típico desses estudos, possivelmente não influiu de maneira relevante nos resultados, uma vez que a maior parte das exposições investigadas refere-se a informações de fácil mensuração (como idade, cor da pele e escolaridade) e/ou exigia apenas o uso da memória recente.

Os controles neste estudo representam a população que deu origem aos casos e que seriam atendidos nesses serviços de saúde se fossem casos. Dessa forma, acredita-se que a comparação entre casos e controles neste estudo é valida e aplica-se à população de mulheres que demandaram os serviços de saúde do inquérito VIVA. Todavia, a possibilidade de viés de seleção devido à escolha dos controles merece reflexão.
Optou-se por escolher controles que também eram vítimas atendidas nos mesmos serviços de saúde e notificados aoVIVA, mas que não fizeram referência à violência doméstica e familiar. Esse tipo de controle pode estar contaminado por casos (como já mencionado) e/ou sobrepareado por algumas exposições relevantes para o tipo de agressão de interesse, levando à subestimação das medidas de efeito. Para minimizar esse tipo de erro na seleção dos controles, optou-se por excluir as vítimas de outros tipos de agressões e as vítimas de lesões autoprovocadas, uma vez que é reconhecido que as mulheres que sofrem violência por parceiro íntimo têm maior risco de desenvolver depressão 18 e que a violência doméstica e familiar pode levar a comportamentos suicidas 19 . Nos Estados Unidos, de 35\% a 40\% das vítimas que sobreviveram à violência doméstica tentaram cometer suicídio em algum momento durante ou após o término da relação abusiva 20.

Em conclusão, a violência doméstica e familiar contra a mulher é um problema complexo e multifatorial. Essas políticas não podem, obviamente, ser restringidas ao setor saúde e devem incluir diversas áreas, como gênero, direitos humanos, justiça, segurança pública, trabalho e previdência, entre outras. Porém, os serviços de saúde têm um papel relevante por serem, em muitos casos, o primeiro contato da vítima com as instituições públicas, que além de procederem o atendimento, devem estar prontos para o acolhimento das vítimas e para ampliar a possibilidade dos passos seguintes no caminho da superação desta condição.

Os sistemas de informação em saúde, assim como os inquéritos realizados no âmbito dos serviços e pesquisas na área da saúde, tais como o VIVA, têm um imenso potencial para a geração de evidências para o conhecimento do tema e subsídios para as políticas públicas voltadas ao enfrentamento da violência contra a mulher.

A violência contra a mulher é passível de prevenção e necessita ser enfrentada. Acreditase que a sua superação implica a eliminação das condições desiguais da mulher na sociedade. Todavia, até que esse ideal seja alcançado, as políticas públicas têm um papel fundamental. É evidente a necessidade de estudos para subsidiar a elaboração e a avaliação dessas políticas, visando ao seu constante aprimoramento. O presente trabalho contribui no sentido de sugerir a adequação dos serviços de atendimento às vítimas, quanto ao preparo para o atendimento e adequação dos períodos de funcionamento, fortalecendo o acesso dessas mulheres, seu acolhimento e a responsabilização por seu cuidado. 


\section{Colaboradores}

L. P. Garcia participou de todas as etapas da elaboração do artigo: concepção, análise, redação e revisão. E. C. Duarte, L. R. S. Freitas e G. D. M. Silva participaram da concepção, redação e revisão da versão final do manuscrito.

\section{Referências}

1. World Health Organization. Global and regional estimates of violence against women: prevalence and health effects of intimate partner violence and non-partner sexual violence. Geneva: World Health Organization; 2013.

2. Schraiber LB, d'Oliveira AFPL, França-Junior I, Diniz S, Portella AP, Ludermir AB, et al. Prevalence of intimate partner violence against women in regions of Brazil. Rev Saúde Pública 2007; 41:797-807.

3. Data Popular; Instituto Patrícia Galvão. Percepção da sociedade sobre violência e assassinatos de mulheres. http://agenciapatriciagalvao.org.br/ wp-content/uploads/2013/08/livro_pesquisa_vio lencia.pdf (acessado em 24/Jan/2015).

4. Instituto Avon; Data Popular. Percepções dos homens sobre a violência doméstica contra a mulher. http://www.compromissoeatitude.org.br/wpcontent/uploads/2013/12/folderpesquisa_institu to22x44_5.pdf (acessado em 25/Jan/2015).

5. Secretaria de Vigilância em Saúde, Ministério da Saúde. Viva: Vigilância de Violências e Acidentes, 2008 e 2009. Brasília: Ministério da Saúde; 2010.

6. Brasil. Lei no 11.340, de 7 de agosto de 2006. Cria mecanismos para coibir a violência doméstica e familiar contra a mulher, nos termos do $\S 8^{\circ}$ do art. 226 da Constituição Federal, da Convenção sobre a Eliminação de Todas as Formas de Discriminação contra as Mulheres e da Convenção Interamericana para Prevenir, Punir e Erradicar a Violência contra a Mulher; dispõe sobre a criação dos Juizados de Violência Doméstica e Familiar contra a Mulher; altera o Código de Processo Penal, o Código Penal e a Lei de Execução Penal; e dá outras providências. Diário Oficial da União 2006; 8 ago.
7. Audi CAF, Segall-Corrêa AM, Santiago SM, Andrade MGG, Pérez-Escamila R. Violence against pregnant women: prevalence and associated factors. Rev Saúde Pública 2008; 42:877-85.

8. d'Oliveira AFPL, Schraiber LB, França-Junior I, Ludermir AB, Portella AP, Diniz CS, et al. Factors associated with intimate partner violence against Brazilian women. Rev Saúde Pública 2009; 43: 299-311.

9. Vieira EM, Perdona GSC, Santos MA. Factors associated with intimate partner physical violence among health service users. Rev Saúde Pública 2011; 45:730-7.

10. Neves ACM, Mascarenhas MDM, Silva MMA, Malta DC. Perfil das vítimas de violências e acidentes atendidas em serviços de urgência e emergência do Sistema Único de Saúde em capitais brasileiras 2011. Epidemiol Serv Saúde 2013; 22:587-96.

11. Kyriacou DN, Anglin D, Taliaferro E, Stone S, Tubb T, Linden JA, et al. Risk factors for injury to women from domestic violence against women. N Engl J Med 1999; 341:1892-8.

12. Sharps PW, Campbell J, Campbell D, Gary F, Webster $\mathrm{D}$. The role of alcohol use in intimate partner femicide. Am J Addict 2001; 10:122-35.

13. Devries RK. Commentary on Devries et al. (2014): a roadmap for future research. Addiction 2014; 109:392-3.

14. Naved RT, Persson LA. Factors associated with spousal physical violence against women in Bangladesh. Stud Fam Plann 2005; 36:289-300.

15. Kishor S, Johnson K. Profiling domestic violence: a multi-country study. Stud Fam Plann 2004; 36: 259-61. 
16. World Health Organization. Responding to intimate partner violence and sexual violence against women: WHO clinical and policy guidelines. Geneva: World Health Organization; 2013.

17. Schlesselman JJ. Case-control studies: design, conduct, analysis. New York: Oxford University Press; 1982. (Monographs in Epidemiology and Biostatistics).

18. Devries K, Watts C, Yoshihama M, Kiss L, Schraiber LB, Deyessa N, et al. Violence against women is strongly associated with suicide attempts: evidence from the WHO multi-country study on women's health and domestic violence against women. Soc Sci Med 2011; 73:79-86.
19. Small Arms Survey. Femicide: a global problem. Research Notes: Armed Violence 2012; (14). http:// www.smallarmssurvey.org/fileadmin/docs/H Research_Notes/SAS-Research-Note-14.pdf (acessado em 24/Jan/2015).

20. Department of Health Statistics and Information Systems, World Health Organization. WHO methods and data sources for global burden of disease estimates 2000-2011. Geneva: World Health Organization; 2013.

\section{Abstract}

This study aimed to identify factors associated with treatment of victims of domestic and family violence in emergency rooms in Brazil. This is a case-control study based on the Surveillance System for Violence and Accidents (VIVA), 2011. Women $\geq 18$ years who were victims of family and domestic violence were selected as cases and compared to accident victims (controls). Adjusted odds ratios were estimated by unconditional logistic regression. 623 cases and 10,120 controls were included. Risk factors according to the adjusted analysis were younger age (18-29 years), low schooling, lack of paid work, alcohol consumption, having sought treatment in a different health service, and violence on weekends or at night or in the early morning hours. The study concludes that domestic and family violence shows alcohol consumption as a strongly associated factor. Days and hours with the highest ocurrence reveal the need to adjust emergency services to treat victims.

Emergency Medical Services; Violence Against Women; Domestic Violence; Women's Health:

Case-Control Studies

\section{Resumen}

El objetivo fue identificar factores asociados a la atención por violencia doméstica y familiar entre víctimas atendidas en servicios de urgencia y emergencia en Brasil. Se realizó un estudio de casos y controles, basado en el Sistema de Vigilancia de Violencia y Accidentes (VIVA), 2011. Se seleccionaron mujeres con 18 años o más de edad, víctimas de violencia doméstica y familiar (casos), en comparación con aquellas víctimas de accidentes (controles). Las razones de momios ajustadas se estimaron por regresión logística no condicional. Se incluyeron 623 casos y 10.120 controles. En el análisis ajustado, fueron factores de riesgo: una edad más joven (18-29 años), baja escolaridad, no ejercer actividad remunerada, consumo de bebidas alcohólicas, búsqueda de atención en otro servicio, incidente acaecido durante el fin de semana y durante la noche o madrugada. La violencia doméstica y familiar tuvo el consumo de bebidas alcohólicas como factor fuertemente asociado. Los días y horas de mayor incidencia evidencian la necesidad de adecuación de los servicios de atención a las víctimas.

Servicios Médicos de Urgencia; Violencia contra la Mujer; Violencia Doméstica; Salud de la Mujer; Estudios de Casos y Controles 